\title{
On the Robustness of Complex Networks by Using the Algebraic Connectivity
}

\author{
A. Jamakovic and P. Van Mieghem \\ Delft University of Technology \\ Electrical Engineering, Mathematics and Computer Science \\ P.O. Box 5031, 2600 GA Delft, The Netherlands \\ A.Jamakovic@ewi.tudelft.nl, P.VanMieghem@ewi.tudelft.nl
}

\begin{abstract}
The second smallest eigenvalue of the Laplacian matrix, also known as the algebraic connectivity, plays a special role for the robustness of networks since it measures the extent to which it is difficult to cut the network into independent components. In this paper we study the behavior of the algebraic connectivity in a well-known complex network model, the Erdős-Rényi random graph. We estimate analytically the mean and the variance of the algebraic connectivity by approximating it with the minimum nodal degree. The resulting estimate improves a known expression for the asymptotic behavior of the algebraic connectivity [18]. Simulations emphasize the accuracy of the analytical estimation, also for small graph sizes. Furthermore, we study the algebraic connectivity in relation to the graph's robustness to node and link failures, i.e. the number of nodes and links that have to be removed in order to disconnect a graph. These two measures are called the node and the link connectivity. Extensive simulations show that the node and the link connectivity converge to a distribution identical to that of the minimal nodal degree, already at small graph sizes. This makes the minimal nodal degree a valuable estimate of the number of nodes or links whose deletion results into disconnected random graph. Moreover, the algebraic connectivity increases with the increasing node and link connectivity, justifies the correctness of our definition that the algebraic connectivity is a measure of the robustness in complex networks.
\end{abstract}

\section{Introduction}

Complex networks describe a wide range of natural and man-made systems, e.g. the Internet, the WWW, networks of food webs, social acquaintances, paper citations, as well as many others [5]11 28]. Although complex systems are extremely different in their function, a proper knowledge of their topology is required to thoroughly understand and predict the overall system performance. For example, in computer networks, performance and scalability of protocols and applications, robustness to different types of perturbations (such as failures and attacks), all depend on the network topology. Consequently, network topology analysis, primarily aiming at non-trivial topological properties, has resulted in the definition of a variety of practically important metrics, capable of quantitatively characterizing certain topological aspects of the studied systems [2|24].

A. Das et al. (Eds.): NETWORKING 2008, LNCS 4982, pp. 183-194 2008.

(C) IFIP International Federation for Information Processing 2008 
In this paper, we rely on a spectral metric, i.e. the second smallest Laplacian eigenvalue, often also referred to as the algebraic connectivity [13]. Fiedler [13] showed that the algebraic connectivity plays a special role: 1) a graph is disconnected if and only if the algebraic connectivity is zero, 2) the multiplicity of zero as an eigenvalue of a graph is equal to the number of disconnected components. There is a vast literature on the algebraic connectivity; see e.g. [9]10 19 20|22] for books and surveys and e.g. [21|23] for applications to several difficult problems in graph theory. However, for the purpose of this work, the most important is its application to the robustness of a graph: 1) the larger the algebraic connectivity is, the more difficult it is to cut a graph into independent components, 2) its classical upper bound in terms of the node and the link connectivity provides worst case robustness to node and link failures [13]. As mentioned in [6], the second means that for every node or link connectivity, there are infinitely many graphs for which the algebraic connectivity is not a sharp lower bound. The node and the link connectivity are important for the robustness because they quantify the extent to which a graph can accommodate to node and link failures. Hence, it is worth investigating the relationship between those three connectivity metrics.

Traditionally, the topology of complex networks has been modeled as Erdős-Rényi random graphs. However, the growing interest in complex networks has prompted many scientists to propose other, more complex models such as "small world" [27] and "scalefree" [4] networks. Despite the fact that various authors have observed that real-world networks have power-law degree distribution, the Erdős-Rényi random graph still has many modeling applications. The modeling of wireless ad-hoc and sensor-networks, peer-to-peer networks like Gnutella [8] and, generally, overlay-networks, provide wellknown examples [14]. Besides that, for the Erdős-Rényi random graph, most of the interesting properties can be analytically expressed. This is in contrast to most other graphs where computations are hardly possible.

Taking the above arguments into consideration, in the first part of this work we study the behavior of algebraic connectivity in the Erdős-Rényi random graph. By using the basic approximation that the algebraic connectivity equals the minimum nodal degree, we estimate the mean and the variance of the algebraic connectivity. Hereby we improve an already existing theorem concerning its behavior [18]. In the second part, we study the relationship between the algebraic connectivity and graph's robustness to node and link failures. Extensive simulations show that the algebraic connectivity increases with the increasing node and link connectivity, implying the correctness of our definition that the algebraic connectivity is a measure of the robustness in complex networks.

The paper is organized as follows. In Section 3, we present the theoretical background on the algebraic connectivity, and the node and the link connectivity. In Section 3 , we analytically derive the estimation of the mean and the variance of the algebraic connectivity for the Erdôs-Rényi model, which we verify by simulations. Prior to analytical derivation, we describe in Section 3.1 the common topological properties that are observed in the random graph of Erdős-Rényi, how they are measured and why they are believed to be important in the context of this paper. In Section 4, we present additional simulation results: by exploring the relation between the algebraic connectivity, and the node and the link connectivity, the existing relations for the connected Erdôs-Rényi graph are refined. Section 5 summarizes our main results. 


\section{Background}

A graph theoretic approach is used to model the topology of a complex system as a network with a collection of nodes $\mathcal{N}$ and a collection of links $\mathcal{L}$ that connect pairs of nodes. A network is represented as an undirected graph $G=(\mathcal{N}, \mathcal{L})$ consisting of $N=|\mathcal{N}|$ nodes and $L=|\mathcal{L}|$ links, respectively. The Laplacian matrix of a graph $G$ with $N$ nodes is an $N \times N$ matrix $Q=\Delta-A$ where $\Delta=\operatorname{diag}\left(D_{i}\right) . D_{i}$ denotes the nodal degree of the node $i \in \mathcal{N}$ and $A$ is the adjacency matrix of $G$.

The eigenvalues of $Q$ are called the Laplacian eigenvalues. The Laplacian eigenvalues $\lambda_{N}=0 \leq \lambda_{N-1} \leq \ldots \leq \lambda_{1}$ are all real and nonnegative [21]. The second smallest Laplacian eigenvalue $\lambda_{N-1}$, also known as the algebraic connectivity, was first studied by Fiedler in [13]. Fiedler showed that the algebraic connectivity is very important for the classical connectivity, a basic measure of the robustness of a graph $G$ : 1) the algebraic connectivity is only equal to zero if $G$ is disconnected, 2) the multiplicity of zero as an eigenvalue of $Q$ is equal to the number of disconnected components of $G$. In [13], Fiedler also proved the following upper bound on the algebraic connectivity $\lambda_{N-1}$ in terms of the minimum nodal degree $D_{\min }$ of a graph $G: 0 \leq \lambda_{N-1} \leq \frac{N}{N-1} D_{\min }$.

In addition, we introduce two connectivity characteristics of a graph $G$ : the link connectivity, i.e. the minimal number of links whose removal results in losing connectivity, is denoted by $\kappa_{L}$ and the node connectivity, which is defined analogously (nodes together with adjacent links are removed) is denoted by $\kappa_{N}$. The following inequality in terms of the node connectivity $\kappa_{N}$ (and obviously the link connectivity $\kappa_{L}$ ) is to be found in [13]: $\lambda_{N-1} \leq \kappa_{N}$. Hence, the minimum nodal degree $D_{\min }$ of an incomplete 1 graph $G$ is an upper bound on both $\lambda_{N-1}$ as well as $\kappa_{N}$ and $\kappa_{L}$. If $\kappa_{N}=\kappa_{L}=D_{\min }$, we say that the connectivity of a graph is optimal.

\section{Algebraic Connectivity in Random Graph of Erdôs-Rényi}

In this section we give an analytical estimate of the algebraic connectivity in the ErdosRényi random graph. The analytical estimate relies on the equality with the minimum nodal degree. This approximation is verified by a comprehensive set of simulations, presented in Subsection 3.4. Prior to analyzing the minimum nodal degree in Subsection 3.2 we give some details on the Erdős-Rényi random graph and the corresponding theorems.

\subsection{Random Graph of Erdôs-Rényi}

The random graph as proposed by Erdős-Rényi [12] is a well-known model to describe a complex network. The most frequently occurring realization of this model is $G_{p}(N)$, where $N$ is the number of nodes and $p$ is the probability of having a link between any two nodes (or shortly the link probability). In fact, $G_{p}(N)$ is the ensemble of all such graphs in which the links are chosen independently and the total number of links is on average equal to $p L_{\max }$, where $L_{\max }=\left(\begin{array}{c}N \\ 2\end{array}\right)$ is the maximum possible number of links.

\footnotetext{
${ }^{1}$ The node connectivity $\kappa_{N}$ of a complete graph $K_{N}$ is $\lambda_{N-1}\left(K_{N}\right)=N>\kappa_{N}\left(K_{N}\right)=N-1$.
} 
Many properties of the random graph can be determined asymptotically, as was shown by Erdôs-Rényi in the series of papers in the 1960s and later by Bollobas in [6]. For example, for a random graph to be connected there must hold, for large $N$, that $p \geq \frac{\log N}{N} \equiv p_{c}$. Moreover, the probability that a random graph for large $N$ is connected, equals $\operatorname{Pr}\left[G_{p}(N)=\right.$ connected $] \simeq e^{-N e^{-p(N-1)}}[26$ ].

Then, the probability that the node connectivity $\kappa_{N}$ equals the link $\kappa_{L}$ connectivity, which in turn equals the minimum nodal degree $D_{\min }$, approaches 1 as $N$ approaches infinity or that $\operatorname{Pr}\left[\kappa_{N}=\kappa_{L}=D_{\text {min }}\right] \rightarrow 1$ as $N \rightarrow \infty$ is also proved in [6] and holds without any restriction on $p$. This was also shown by Bollobás and Thomason in [7]. On the other hand, the asymptotic behavior of the algebraic connectivity in the Erdôs-Rényi random graph $G_{p}(N)$ is proved by Juhāz in [18]: For any $\varepsilon>0$,

$$
\lambda_{N-1}=p N+o\left(N^{\frac{1}{2}+\varepsilon}\right)
$$

where the algebraic connectivity converges in probability as $N \rightarrow \infty$.

\subsection{Minimum Nodal Degree in Random Graph of Erdős-Rényi}

In $G_{p}(N)$ each node $i$ has a degree $D_{i}$ that is binomially distributed. Before proceeding, we first need to show that degrees in the sequence $\left\{D_{i}\right\}_{1 \leq i \leq N}$ are almost independent random variables. In any graph $\sum_{i=1}^{N} D_{i}=2 L$ holds, thus degrees in the sequence $\left\{D_{i}\right\}_{1 \leq i \leq N}$ are not independent. However, if $N$ is large enough, $D_{i}$ and $D_{j}$ are almost independent for $i \neq j$ and we can assume that all $D_{i}$ are almost i.i.d. binomially distributed (see also [6, p. 60]). The following Lemma quantifies this weak dependence:

Lemma 1. The correlation coefficient of the degree $D_{i}$ and $D_{j}$ of two random nodes $i$ and $j$ in $G_{p}(N)$ for $0<p<1$ is

$$
\rho\left(D_{i}, D_{j}\right)=\frac{\operatorname{Cov}\left[D_{i}, D_{j}\right]}{\sqrt{\operatorname{Var}\left[D_{i}\right]} \sqrt{\operatorname{Var}\left[D_{j}\right]}}=\frac{1}{N-1} .
$$

Proof. See Appendix A of [16]. For large $N$ and constant $p$, independent of $N$, the normalized i.i.d. binomially distributed sequence $\left\{D_{i}^{*}\right\}_{1 \leq i \leq N}$ of all degrees in $G_{p}(N)$ tends to be Gaussian distributed. The minimum of the sequence $\left\{D_{i}^{*}\right\}_{1<i<N}$ possesses the distribution

$$
\operatorname{Pr}\left[\min _{1 \leq i \leq N} D_{i}^{*} \leq x\right]=1-\prod_{i=1}^{N} \operatorname{Pr}\left[D_{i}^{*}>x\right]=1-\left(\operatorname{Pr}\left[D_{i}^{*}>x\right]\right)^{N} .
$$

After considering the limiting process of the minimum of a set $\left\{D_{i}^{*}\right\}_{1 \leq i \leq N}$ when $N \rightarrow \infty$, we derive [16] the appropriate solution

$$
D_{\min }^{*}=\frac{-Y-2 \log N+\log \left(\sqrt{2 \pi \log \frac{N^{2}}{2 \pi}}\right)}{\sqrt{2 \log N}}
$$


where $Y$ is a Gumbel random variable [26]. With $D_{\min }=\sigma[D] \cdot D_{\min }^{*}+E[D]=$ $\sqrt{(N-1) p(1-p)} \cdot D_{\min }^{*}+p(N-1)$, we obtain

$$
D_{\min }=p(N-1)-\sqrt{(N-1) p(1-p)}\left(\frac{Y+2 \log N-\log \left(\sqrt{2 \pi \log \frac{N^{2}}{2 \pi}}\right)}{\sqrt{2 \log N}}\right) .
$$

Finally, let $D_{\min }(p)$ denote the minimum degree in $G_{p}(N)$. Since the complement of $G_{p}(N)$ is $G_{1-p}(N)$, there holds that

$$
D_{\min }(p)=N-1-D_{\max }(1-p) .
$$

The law of $D_{\max }$ has been derived by Bollobas [6, Corollary 3.4 (p. 65)] via another method. Using the above relation, Bollobas' results precisely agrees with ours.

\subsection{Analytical Approximation for Algebraic Connectivity in Random Graph of Erdôs-Rényi}

In Section 2 we saw that $\lambda_{N-1} \leq \frac{N}{N-1} D_{\min }$. Our basic approximation is $\lambda_{N-1} \simeq$ $D_{\text {min }}$ for large $N$. A comprehensive set of simulation results, presented in Subsection 3.4 supports the quality of this assumption. With this approximation we arrive, for large $N$, at

$$
\begin{aligned}
\lambda_{N-1} \simeq & p(N-1)-\sqrt{2 p(1-p)(N-1) \log N} \\
& +\sqrt{\frac{(N-1) p(1-p)}{2 \log N}} \log \left(\sqrt{2 \pi \log \frac{N^{2}}{2 \pi}}\right) \\
& -\sqrt{\frac{(N-1) p(1-p)}{2 \log N}} Y .
\end{aligned}
$$

By taking the expectation on both sides and taking into account that the mean of a Gumbel random variable $E[Y]=\gamma=0.5772 \ldots$, our estimate of the mean of the algebraic connectivity in $G_{p}(N)$ becomes, for large $N$ and constant $p$,

$$
\begin{aligned}
E\left[\lambda_{N-1}\right] \simeq & p(N-1)-\sqrt{2 p(1-p)(N-1) \log N} \\
& +\sqrt{\frac{(N-1) p(1-p)}{2 \log N}} \log \left(\sqrt{2 \pi \log \frac{N^{2}}{2 \pi}}\right) \\
& -\sqrt{\frac{(N-1) p(1-p)}{2 \log N}} \gamma
\end{aligned}
$$

Similarly, by taking into account that $\operatorname{Var}[Y]=\frac{\pi^{2}}{6}$, the estimate of the variance of the algebraic connectivity in $G_{p}(N)$ is

$$
\operatorname{Var}\left[\lambda_{N-1}\right]=\frac{(N-1) p(1-p)}{2 \log N} \frac{\pi^{2}}{6} .
$$




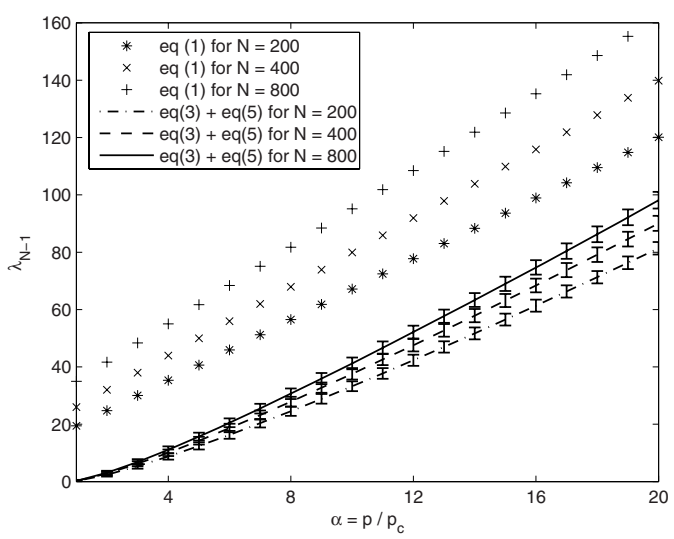

Fig. 1. A comparison between the estimation of the mean (3) as well as the standard deviation (5), plotted in lines and error bars, and the theorem of Juhāz (1), plotted in markers, for the algebraic connectivity $\lambda_{N-1}$ as a function of $\alpha=p / p_{c}$ in the Erdős-Rényi random graph $G_{p}(N)$ with $N=200,400$ and 800 nodes

An interesting observation is that the standard deviation

$$
\sigma\left[\lambda_{N-1}\right]=\sqrt{\operatorname{Var}\left[\lambda_{N-1}\right]}=O\left(\sqrt{\frac{N}{\log N}}\right)
$$

is much smaller than the mean (3). This implies that $\lambda_{N-1}$ tends to the mean rapidly, or that, for large $N, \lambda_{N-1}$ behaves almost deterministically and is closely approximated by the first three terms in (2). Hence, the relation (2) is more accurate than (1) (see also Figure 10.

\subsection{Verification of Analytical Approximation by Simulations}

In all simulations we consider exclusively the Erdôs-Rényi random graph $G_{p}(N)$ with various combinations of the number of nodes $N$ and the link probabilities $p . N$ can takes the following values: 200,400 and 800 . The link probability $p=\alpha p_{c}=\alpha \frac{\log N}{N}$, where $\alpha$ is varying from 1 to 20. From each combination of $N$ and $p$, we compute the algebraic connectivity $\lambda_{N-1}$ and the minimum nodal degree $D_{\min }$. Then, we classify the simulated graphs according to their value of $\alpha$, as shown in Figures 2 and 3 . Subsequently, from generated graphs with a given $\alpha$, we are interested in the extreme values, i.e. $\min \lambda_{N-1}$ and $\max \lambda_{N-1}$, as shown in Figures 4 and 5 .

In Figures 2 and 3 , we have plotted the simulated mean $E\left[\lambda_{N-1}\right]$, the corresponding standard deviation $\sigma\left[\lambda_{N-1}\right]$, and our estimate for the mean, Eq. (3), and the standard deviation, Eq. (5), of the algebraic connectivity as a function of $\alpha$. As illustrated in Figures in Figures 2 and 3 there is a remarkable correspondence between the simulations and our estimate: the standard deviation is much smaller than the mean, implying that for $N \rightarrow \infty, \lambda_{N-1}$ will rapidly approach $E\left[\lambda_{N-1}\right]$. Moreover, our basic approximation that, for large $N, \lambda_{N-1} \simeq D_{\min }$ is verified by the simulations shown in Figures 4 and 5 
We found that $\min \lambda_{N-1}$ or $\max \lambda_{N-1}$ grows linearly with $D_{\min }$. Note in Figure 4 that, in the probability range around the connectivity threshold $p_{c}$, the minimum algebraic connectivity is always equal to zero, indicating a non-connected random graph (for details see Section 4).

From Figures 4 and 5 it is clear that if the value of the algebraic connectivity is larger than zero, the random graph has nodes of minimum degree always larger than zero too, referring to $\left\{\lambda_{N-1}>0\right\} \Longleftrightarrow\left\{G_{p}(N)\right.$ is connected $\}$. However, by scrutinizing only degree-related simulation results, we see that the implication $\left\{D_{\min } \geq 1\right\} \Longrightarrow$ $\left\{G_{p}(N)\right.$ is connected $\}$ is not always true, i.e. for large $N$ and certain $p$ which depends on $N$, the implication is almost surely (a.s.) correct [26]. For example, the percentage of graphs with $D_{\min } \geq 1$ that leads to a connected $G_{p}(50)$ increases from $98 \%$ for $p=p_{c}$ to $100 \%$ for $2 p_{c}$, while the percentage for $G_{p}(400)$ increases from $99 \%$ for $p=p_{c}$ to $100 \%$ for $2 p_{c}$. Hence, the simulation results confirm that, for large $N$ and rather small $p=\frac{\log N}{N}$, the latter implication a.s. is equivalent.

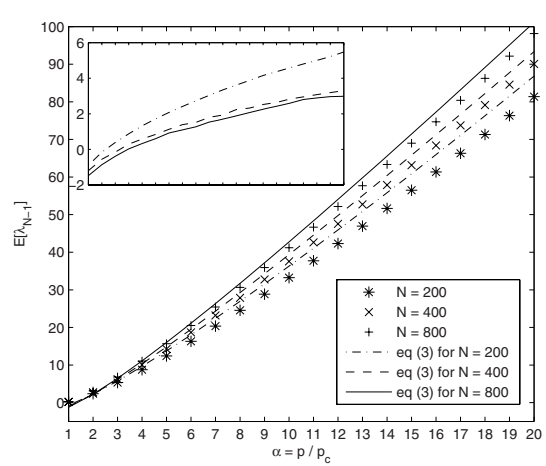

Fig. 2. A comparison between the estimation (3), plotted in lines, and the simulation results, plotterd in markers, for the mean of the algebraic connectivity $E\left[\lambda_{N-1}\right]$ as a function of $\alpha$. In the upper left corner of the figure, we show the difference between the estimation and the simulation results as a function of $\alpha$.

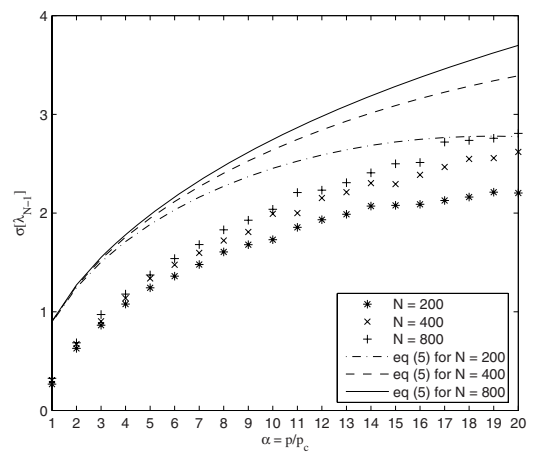

Fig. 3. A comparison between the estimation (5), plotted in lines, and the simulation results, plotterd in markers, for the standard deviation of the algebraic connectivity $\sigma\left[\lambda_{N-1}\right]$ as a function of $\alpha$

Simulations demonstrate also that, for a particular fixed $\alpha=\frac{p}{p_{c}}$, the mean of the algebraic connectivity increases with the size of the random graph: a higher value of the graph size $N$ implies a higher mean of the algebraic connectivity, what in turn indicates that the probability of having a more robust graph is approaching 1 as $N \rightarrow$ $\infty$. Theorem given in Subsection 3.1 stating that $\operatorname{Pr}\left[\kappa_{N}=\kappa_{L}=D_{\text {min }}\right] \rightarrow 1$ as $N \rightarrow \infty$, clarifies this observation in a slightly different way: given that $N$ is approaching $\infty$, the node and the link connectivity will become as high as possible, i.e. equal to the minimum nodal degree, and therefore the graph will become optimally connected. 


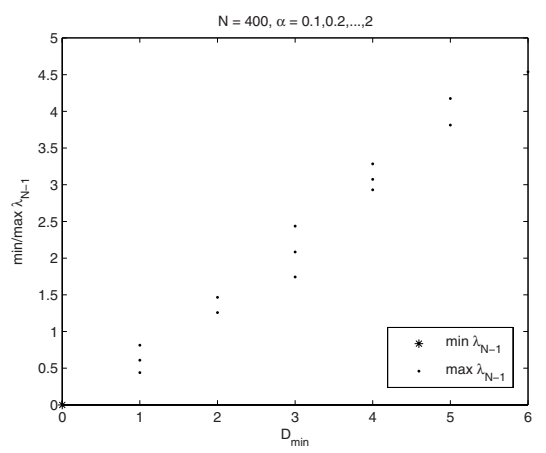

Fig. 4. The relationship between the minimum algebraic connectivity $\min \lambda_{N-1}$ (maximum algebraic connectivity $\left.\max \lambda_{N-1}\right)$ and the minimum nodal degree $D_{\min }$ in the Erdôs-Rényi random graph $G_{p}(N)$. For each combination of $N$ and $p=\alpha p_{c}, \alpha=0.1,0.2,0.3, \ldots, 2$, we generate $10^{4}$ random graphs. Then, from the generated graphs, having a given value of $\alpha$, we take $\min \lambda_{N-1}\left(\max \lambda_{N-1}\right)$ and the corresponding $D_{\text {min }}$, resulting in one point for each considered $\alpha$.

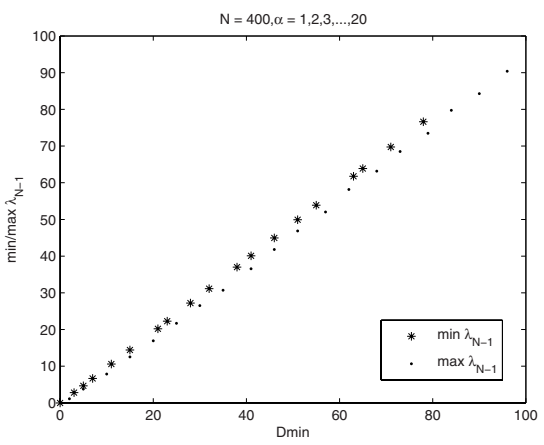

Fig. 5. The relationship between the minimum algebraic connectivity $\min \lambda_{N-1}$ (maximum algebraic connectivity $\left.\max \lambda_{N-1}\right)$ and the minimum nodal degree $D_{\min }$ in the Erdôs-Rényi random graph $G_{p}(N)$. For each combination of $N$ and $p=\alpha p_{c}, \alpha=1,2,3, \ldots, 20$, we generate $10^{4}$ random graphs. Then, from the generated graphs, having a given value of $\alpha$, we take $\min \lambda_{N-1}\left(\max \lambda_{N-1}\right)$ and the corresponding $D_{\min }$, resulting in one point for each considered $\alpha$.

\section{Relationship between Algebraic, Node and Link Connectivity in Random Graph of Erdôs-Rényi}

In the previous section we analytically estimated the behavior of the algebraic connectivity in the Erdős-Rényi random graph. In this section we analyze the relation among the three connectivity measures: the algebraic connectivity, the node connectivity and the link connectivity.

We have used the polynomial time algorithm, explained in [15], to find the node and the link connectivity by solving the maximum-flow problem. The maximum-flow problem can be solved with several algorithms, e.g. Dinic, Edmonds \& Karp, Goldberg, etc. If Goldberg's push-relabel algorithm is utilized, as performed in our simulations, the link connectivity algorithm has $O\left(N^{3} \sqrt{L}\right)$-complexity, while the node connectivity algorithm has $O\left(N^{2} L \sqrt{L}\right)$-complexity. We have used the LAPACK implementation of the QR-algorithm for computing all the eigenvalues of the Laplacian matrix. For linear algebra problems involving the computation of a few extreme eigenvalues of large symmetric matrices, algorithms (e.g. Lanczos) whose run-time and storage cost is lower compared to the algorithms for calculation of all eigenvalues (QR algorithm has $O\left(n^{3}\right)$-complexity) are known [3].

We simulate for each combination of $N$ and $p, 10^{4}$ independent $G_{p}(N)$ graphs. $N$ is 50,100, 200 and 400 nodes and the link probability $p=\alpha p_{c}$, where $\alpha$ varies from 1 to 10 . From each combination of $N$ and $p$, we compute the minimum nodal degree 


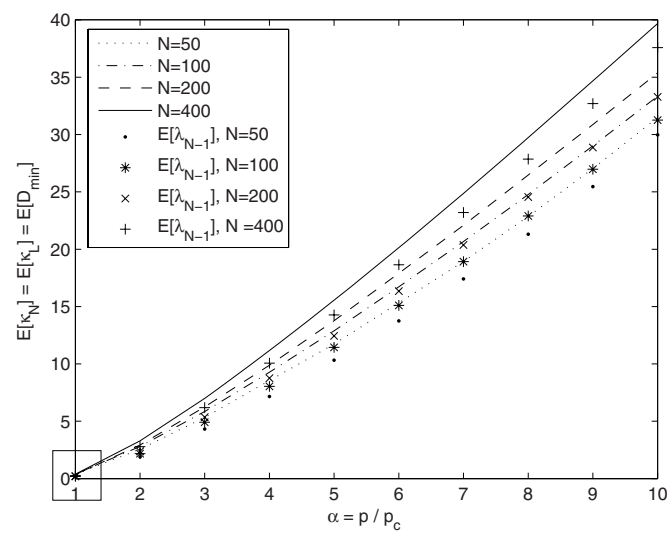

Fig. 6. Simulated results on Erdős-Rényi random graph $G_{p}(N)$ for $N=50,100,200,400$ and the link probability $p=\alpha p_{c}$, showing the mean of the algebraic connectivity $E\left[\lambda_{N-1}\right]$, the mean of the node $E\left[\kappa_{N}\right]$ and the link $E\left[\kappa_{L}\right]$ connectivity and the mean of the minimum nodal degree $E\left[D_{\min }\right]$ as a function of $\alpha$, where $\alpha=1,2, \ldots, 10$. Note that for $\alpha=1, E\left[\kappa_{N}\right]=E\left[\kappa_{L}\right]$ but $E\left[\kappa_{N}\right] \neq E\left[D_{\min }\right]$.

$D_{\text {min }}$, the algebraic, the node and the link connectivity, denoted respectively by $\lambda_{N-1}$, $\kappa_{N}$ and $\kappa_{L}$. Then, we classify graphs according to their value of $\alpha$.

Figure 6 shows the mean value of the algebraic connectivity $E\left[\lambda_{N-1}\right]$ as a function of increasing $\alpha=\frac{p}{p_{c}}$. In addition, Figure 6 shows the mean of the node connectivity $E\left[\kappa_{N}\right]$, the link connectivity $E\left[\kappa_{L}\right]$ and the minimum nodal degree $E\left[D_{\min }\right]$.

The first conclusion we can draw after analyzing simulation data is that for all generated random graphs from $p=p_{c}$ to $p=10 p_{c}$ the convergence to a surely connected random graph, i.e. $\lambda_{N-1}>0$, is surprisingly rapid. Results concerning connectivity percentages are plotted in Figure 7 For example, the percentage of connected random graphs with 50 nodes increases from about $39 \%$ and $98 \%$ for $p_{c}$ and $p=2 p_{c}$, respectively, to $99 \%$ for $p=3 p_{c}$, where for $p=4 p_{c}$ the graph is connected. These results are consistent with the Erdős-Rényi asymptotic expression. For $N \rightarrow \infty$, as observable in Figure 7 the simulated data as well as the Erdős-Rényi formula confirm a well known result [17] that the random graph $G_{p}(N)$ is a.s. disconnected if the link density $p$ is below the connectivity threshold $p_{c} \sim \frac{\log N}{N}$ and connected for $p>p_{c}$.

The second conclusion is that our results, regarding the distribution range of the algebraic connectivity and the minimum nodal degree, indeed comply with the bounds $0 \leq \lambda_{N-1} \leq \frac{N}{N-1} D_{\text {min }}$ : the distribution of the algebraic connectivity $\lambda_{N-1}$ is contained in the closed interval $[0, N]$, or to be more precise $\lambda_{N-1}$ is 0 for a disconnected graph and above bounded by $\frac{N}{N-1} D_{\min }$ for all those link probabilities $p$ for which the graph is connected but not complete 2 . Then, obviously $E\left[\lambda_{N-1}\right] \leq E\left[D_{\min }\right]$.

The third conclusion is that the distribution range of the algebraic connectivity also complies with the bounds $\lambda_{N-1} \leq \kappa_{N}$. Moreover, in Figure 6, for $p>p_{c}$ and all simulated $N$, the distributions of the node $\kappa_{N}$ and the link $\kappa_{L}$ connectivity are equal to

\footnotetext{
${ }^{2}$ If a graph $G$ is a complete graph $K_{N}$ then $\lambda_{N-1}=N>D_{\min }=N-1$.
} 
the distribution of the minimum nodal degree $D_{\min }$ (recall that in Figure 6 for $p=p_{c}$, the distributions of $\kappa_{N}, \kappa_{L}$ and $D_{\min }$ are almost equal but not the same). Convergence here to a graph where $\kappa_{N}=\kappa_{L}=D_{\text {min }}$ is surprisingly rapid. For example, from the simulation results plotted in Figure 8 with $p=p_{c}$ and size of the random graph ranging from $N=5$ to $N=400$, we found that with probability approaching 1 , the random graph becomes optimally connected at rather small graph sizes. For all other link probabilities, $p>p_{c}$, the convergence to $\kappa_{N}=\kappa_{L}=D_{\min }$ is faster (see Figure 8 for $p=2 p_{c}$ ). Hence, the simulation results show that the random graph $G_{p}(N)$ a.s. is constructed in such a way that deleting all the neighbors (or the links to its neighbors) of a minimum nodal degree node will lead to the minimum number of nodes (links) whose deletion from a graph will result into a disconnected random graph. Hence, the minimum nodal degree is a valuable estimate of the number of nodes or links whose deletion results into a disconnected graph.

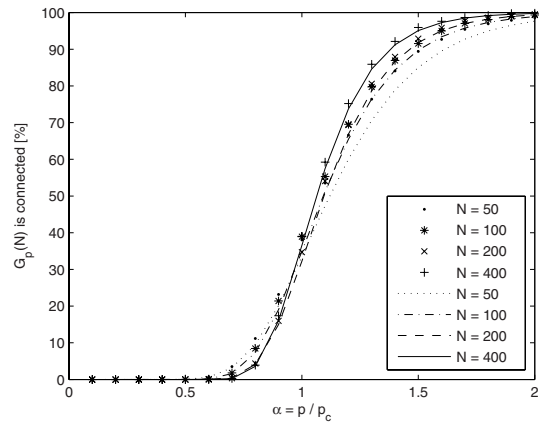

Fig. 7. Percentage of the connected Erdős-Rényi random graphs $G_{p}(N)$, i.e. $\lambda_{N-1}>0$ : a comparison between the simulation results, plotted in markers, and the Erdos' asymptotic formula $\operatorname{Pr}\left[G_{p}(N)=\right.$ connected $] \simeq e^{-N e^{-p(N-1)}}$, plotted in lines, in the probability range $\alpha=p / p_{c}=0.1,0.2, \ldots, 2$

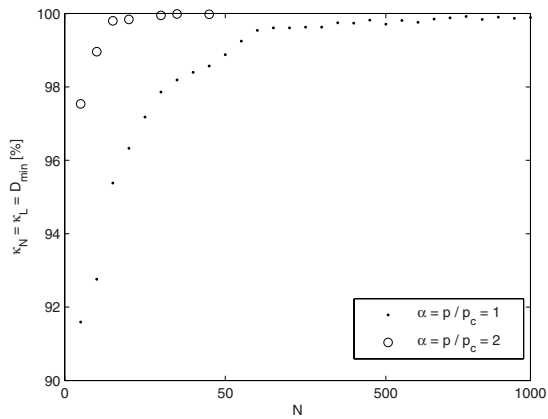

Fig. 8. Percentage of the Erdős-Rényi random graphs $G_{p}(N)$ with $p=p_{c}$ and $p=2 p_{c}$ in which the node connectivity $\kappa_{N}$, the link connectivity $\kappa_{L}$ and the minimum nodal degree $D_{\text {min }}$ converge to $\kappa_{N}=\kappa_{L}=D_{\min }$ for small graph sizes $N$

\section{Conclusion}

We studied the algebraic connectivity and its relation to the node and the link connectivity in the Erdős-Rényi random graph. The analytical study shows that the variance of the algebraic connectivity is much smaller than its mean, implying that, for large graph size $N$, the distribution of the algebraic connectivity will rapidly approach the mean value. Through extensive simulations, we verified that the algebraic connectivity behaves almost deterministically and is closely approximated by our basic estimate, Eq. (2). Simulations also show that, for large $N$, the distribution of the algebraic connectivity grows linearly with the minimum nodal degree, confirming our basic approximation that $\lambda_{N-1} \simeq D_{\min }$. 
Moreover, for a given value of $\alpha=\frac{p}{p_{c}}$, a higher value of the graph size $N$ means a higher value of the algebraic connectivity. This translates into a higher probability of having a more connected, or to say robust, graph as $N \rightarrow \infty$. On the other hand, the larger the graph size, the more the Erdős-Rényi random graph is constructed in such a way that deleting all the neighbors (or the links to its neighbors) of a minimum nodal degree node leads to the minimum number of nodes (links) whose deletion disconnects the graph. However, the simulation results show that this optimal connectivity, occurs, regardless of the link probability $p$, at already small graph sizes $N$. Hence, the larger the value of the algebraic connectivity, the better the graph's robustness to node and link failures.

\section{References}

1. Abramowitz, M., Stegun, I.A.: Handbook of Mathematical Functions. Dover Publications, Inc, NY (1999)

2. Albert, R., Barabasi, A.-L.: Statistical mechanics of complex networks. Reviews of Modern Physics 74(1) (January 2002)

3. Bai, Z., Demmel, J., Dongarra, J., Ruhe, A., van der Vorst, H.: Templates for the solution of Algebraic Eigenvalue Problems: A Practical Guide. SIAM, Philadelphia (2000)

4. Barabasi, A.-L., Albert, R.: Emergence of scaling in random networks. Science 286, 509-512 (1999)

5. Barabasi, A.-L.: Linked. The new science of networks. Perseus, Cambridge (2002)

6. Bollobás, B.: Random graphs, 2nd edn. Cambridge University Press, Cambridge (2001)

7. Bollobás, B., Thomason, A.G.: Random graphs of small order. Random graphs 1983. Annals of Discrete Mathematics 28, 47-97 (1985)

8. Castro, M., Costa, M., Rowstron, A.: Should We Build Gnutella on a Structured Overlay. ACM SIGCOMM Computer Communications Review 34(1), 131-136 (2004)

9. Chung, F.R.K.: Spectral Graph Theory. In: Conference Board of the Mathematical Sciences, American Mathematical Society, Providence, RI, vol. 92 (1997)

10. Cvetkovic, D.M., Doob, M., Sachs, H.: Spectra of Graphs, Theory and Applications, 3rd edn. Johann Ambrosius Barth, Heidelberg (1995)

11. Dorogovtsev, S.N., Mendes, J.F.F.: Evolution of Networks. In: From Biological Nets to the Internet and WWW, Oxford University Press, Oxford (2003)

12. Erdős, P., Rényi, A.: On random graphs. Publicationes Mathematicae 6, 290-297 (1959)

13. Fiedler, M.: Algebraic connectivity of graphs. Czechoslovak Mathematical Journal 23, 298 305 (1973)

14. Fuhrmann, T.: On the Topology of Overlay Networks. In: Proceedings of 11th IEEE International Conference on Networks (ICON), pp. 271-276 (2003)

15. Gibbons, A.: Algorithmic Graph Theory. Cambridge University Press, Cambridge (1985)

16. Jamakovic, A., Van Mieghem, P.: On the robustness of complex networks by using the algebraic connectivity, Technical Report (2007)

17. Janson, S., Knuth, D.E., Luczak, T., Pittel, B.: The birth of the giant component. Random Structures \& Algorithms 4, 233-358 (1993)

18. Juhāz, F.: The asymptotic behaviour of Fiedler's algebraic connectivity for random graphs. Discrete Mathematics 96, 59-63 (1991)

19. Merris, R.: Laplacian matrices of graphs: a survey. In: Linear Algebra Applications, vol. 197,198 , pp. 143-176 (1994)

20. Merris, R.: A survey of graph Laplacians. In: Linear and Multilinear Algebra, vol. 39, pp. 19-31 (1995) 
21. Mohar, B., Alavi, Y., Chartrand, G., Oellermann, O.R., Schwenk, A.J.: The Laplacian spectrum of graphs. Graph Theory, Combinatorics and Applications 2, 871-898 (1991)

22. Mohar, B.: Laplace eigenvalues of graphs: a survey. Discrete Mathematics 109, 198, 171-183 (1992)

23. Mohar, B., Hahn, G., Sabidussi, G.: Some applications of Laplace eigenvalues of graphs. Graph Symmetry: Algebraic Methods and Applications, NATO ASI Ser. C 497, 225-275 (1997)

24. Newman, M.: The structure and function of complex networks. SIAM Review 45, 167-256 (1990)

25. Skiena, S.: Implementing Discrete Mathematics: Combinatorics and Graph Theory with Mathematica. Addison-Wesley, Reading (1990)

26. Van Mieghem, P.: Performance Analysis of Computer Systems and Networks. Cambridge University Press, Cambridge (2006)

27. Watts, D.J., Strogatz, S.H.: Collective dynamics of small-world networks. Nature 393, 440 442 (1999)

28. Watts, D.J.: Small Worlds. The Dynamics of Networks Between Order and Randomness. Princeton University Press, Princeton (1999) 